\title{
Propiedades Físicas y Mecánicas de Bloques de Hormigón Compuestos con Áridos Reciclados
}

\author{
Gonzalo A. Valdés y Jorge G. Rapimán \\ Departamento de Ingeniería de Obras Civiles, Universidad de la Frontera, \\ Francisco Salazar No 01145, Temuco-Chile
}

\begin{abstract}
Resumen
El principal objetivo que tiene este artículo es mostrar una técnica para la confección de bloques prefabricados de hormigón utilizando árido reciclado proveniente de demoliciones de pavimentos y verificar el grado de cumplimiento de las normativas actuales que rigen la elaboración de estos elementos de construcción. Se analizaron las características físicas y granulométricas del árido constituyente de la mezcla, y se dosificó el cemento y áridos de los hormigones de prueba de acuerdo al método Faury-Joisel. Los áridos estudiados mostraron diferencias en las densidades, siendo menores las del material reciclado, pero siempre dentro de los márgenes establecidos en la normativa. Los ensayos a la compresión efectuados a bloques de hormigón confeccionados con material reciclado muestran resistencias inferiores en un $15 \%$ a los confeccionados con áridos naturales. Sin embargo, ambos elementos cumplieron con los parámetros establecidos en la normativa vigente para la resistencia mecánica a la compresión, la absorción de máxima de agua y el contenido de humedad.
\end{abstract}

Palabras clave: bloques prefabricados, árido reciclado, residuos de hormigón, construcción

\section{Physical and Mechanical Properties of Concrete Bricks Produced with Recycled Aggregates}

\begin{abstract}
The main objective of this paper is to show a technique for the construction of prefabricated concrete bricks utilizing recycled aggregates coming from demolitions of pavements and to check the degree of fulfillment of the current rules that are applied to the manufacture of these building elements. The physical and grain-size characteristics of the aggregates constituent of the mixture were analyzed, and the cement and aggregates were dozed according to the Faury-Joisel method. The analyzed aggregates showed differences in densities being lower the ones made of recycled material, but always within the established limits of the current norms. The compression tests were carried out on the concrete bricks manufactured with recycled material showed resistances of $15 \%$ lower than those manufactured with natural aggregates. However, both elements fulfilled with the parameters established by the current norm for the mechanical resistance, the maximum water absorption and the humidity content.
\end{abstract}

Keywords: concrete bricks, recycled aggregates, waste concrete, building materials 


\section{INTRODUCCION}

Una gran cantidad de escombros producto de la demolición de estructuras son producidos anualmente en los países desarrollados, en que los depósitos de escombros derivados de la actividad de la construcción, ha llegado a ser un serio problema social y ambiental para las ciudades, debido a la necesidad de disponer terrenos para su vertimiento, como también el alto costo que implica su manejo. La necesidad de reciclar los escombros que produce la industria de la construcción está tomando, hoy en día, gran importancia (Poon et al., 2002). Estudios realizados en Hong Kong muestran que un 55\% de la composición de los escombros generados en la construcción son hormigón sin armar y hormigón armado (Poon et al., 2001), situación genera una gran cantidad de materia prima para ser reutilizada en nuevas estructuras. En Chile, el problema es similar, aunque no existen datos precisos sobre la producción de residuos generados por la actividad de la construcción; la Corporación Nacional del Medio Ambiente estima que se generaran alrededor de 26.000 m3 de escombros solamente en la IX región de Chile (Montoya et al., 2005).

Otro punto importante a considerar es la reducción de extracción de materiales pétreos de los entornos naturales, disminuyendo el impacto ambiental y el rápido agotamiento de las reservas naturales de áridos provenientes tanto de cauces como de canteras (Rakshvir y Barai, 2006). La gran demanda de recursos básicos ha generado una gran escasez de materias primas, constituyendo una gran preocupación, sumando a esto la motivación que existe de preservar y proteger el medio ambiente de un inminente desequilibrio ecológico (Aguilar et al., 2005). El Hormigón como material de construcción requiere un volumen importante de recursos, especialmente agregados pétreos. En los últimos años, estudios nacionales e internacionales han demostrado que las propiedades físicas y mecánicas del hormigón constituido por adiciones racionales de árido reciclado en su mezcla garantiza la utilización adecuada de este material (Aguilar et al., 2005; Montoya et al.,2005; Rakshvir y Barai, 2006; Sánchez y Alaejos, 2006; Topcu, 1997; Topcu y Guncan, 1995; Topcu y Sengel, 2004).

El presente trabajo busca verificar que los bloques de hormigón elaborados con árido grueso reciclado, arena natural y cemento de alta resistencia inicial cumplan las actuales normas que rigen la confección y utilización de este elemento en Chile (NCh 181 of 67), además de realizar una comparación con bloques elaborados completamente áridos naturales diseñados y construidos bajo las mismas condiciones.

\section{MATERIALES}

Cemento, áridos naturales y áridos reciclados.

El material cementicio utilizado correspondió a un cemento de alta resistencia inicial elaborado sobre la base de clinker, escoria básica granulada de alto horno y yeso. De acuerdo a la NCh 148 of.68, se clasifica según su composición y resistencia como cemento Portland siderúrgico, grado alta resistencia. Según la norma ASTM C-595 (USA), se clasifica como Slag Modified Portland Cement. Según la Norma BS EN 197-1:2000, notación II/A-S. Las características técnicas del cemento utilizado se ilustran en la Tabla 1.

El árido natural utilizado, fino y grueso, corresponde a un material procesado, obtenido de lechos y ribera de cauces naturales, compuestos principalmente por partículas de dolomita, basalto, dacitas, andesitas, riolitas, arenisca, cuarzo y cuarcita (Moreno et al.1985). El tamaño máximo nominal del árido grueso utilizado en este estudio fue de $12.5 \mathrm{~mm}$. Las características granulométricas y propiedades físicas del árido grueso natural utilizado se muestran en las Tablas 2 y 3, mientras que las características granulométricas y propiedades físicas del árido fino natural se ilustran en las Tablas 4 y 5.

El árido reciclado utilizado en este trabajo se obtuvo de residuos de estructuras hormigón, principalmente de demoliciones de pavimentos, situadas en un botadero en ribera del río Cautín, IX región de Chile. Las estructuras de hormigón desechadas fueron pretrituradas en laboratorio con un martillo neumático con la finalidad de disminuir su tamaño a trozos de 10", posteriormente los trozos 
de hormigón se procesaron en una planta chancadora y se seleccionaron por medio de tamices, obteniéndose un material de tamaño máximo nominal de 3", los cuales fueron seleccionados en 12,5 $\mathrm{mm}$, tamaño máximo utilizado en este estudio. Se consideró solo la fracción gruesa del árido reciclado dentro de la mezcla utilizada. Las características granulométricas y propiedades físicas del árido grueso reciclado utilizado, se muestran en las Tablas 6 y 7.

Tabla 1. Características técnicas del cemento utilizado

\begin{tabular}{|l|l|}
\hline Propiedades & Portland Siderúrgico \\
\hline Clase & De alta resistencia \\
\hline Grado & Características Físicas y Mecánicas \\
\hline Peso específico (g/cm $\left.{ }^{3}\right)$ & 3.0 \\
\hline Expansión autoclave (\%) & 0.05 \\
\hline Fraguado inicial (h:m) & $02: 00$ \\
\hline Fraguado final (h:m) & $02: 40$ \\
\hline Resistencia a la compresión NCh 158 \\
\hline 3 días & 300 \\
\hline 7 días & 400 \\
\hline 28 días & 520 \\
\hline 90 días & 620 \\
\hline Características Químicas \\
\hline Perdidas por calcinación (\%) & 2.0 \\
\hline SO3 (\%) & 2.4 \\
\hline
\end{tabular}

Tabla 2. Características granulométricas árido grueso natural

\begin{tabular}{|c|c|c|}
\hline ASTM No & $\mathrm{mm}$ & Porcentaje en peso que pasa \\
\hline $1 / 2 "$ & 12.7 & 100 \\
\hline $3 / 8 "$ & 9.51 & 39.5 \\
\hline 4 & 4.76 & 5.2 \\
\hline 8 & 2.38 & 3.2 \\
\hline
\end{tabular}

Tabla 3. Características físicas del árido grueso natural

\begin{tabular}{|l|c|}
\hline \multicolumn{2}{|l|}{ Propiedades } \\
\hline Densidad aparente compactada $\left(\mathrm{Kg} / \mathrm{dm}^{3}\right)$ & 1.58 \\
\hline Densidad aparente suelta $\left(\mathrm{Kg} / \mathrm{dm}^{3}\right)$ & 1.47 \\
\hline Densidad real seca $\left(\mathrm{Kg} / \mathrm{dm}^{3}\right)$ & 2.66 \\
\hline Densidad real saturada sup. seca $\left(\mathrm{Kg} / \mathrm{dm}^{3}\right)$ & 2.7 \\
\hline Densidad neta $\left(\mathrm{Kg} / \mathrm{dm}^{3}\right)$ & 2.76 \\
\hline Absorción $(\%)$ & 1.43 \\
\hline Partículas menores a $0.08 \mathrm{~mm} \mathrm{( \% )}$ & 0.08 \\
\hline
\end{tabular}

Las características granulométricas y propiedades físicas de los áridos naturales finos y gruesos, y el árido reciclado grueso, fueron determinadas de acuerdo a las normas chilenas del Instituto Nacional de Normalización, NCh 163 Of 79, NCh 1116 EOf 77, NCh 1117 EOf 77, NCh 1223 EOf 77, NCh 1239 Of77 y NCh 166 Of 52. 
Tabla 4. Características granulométricas árido fino natural

\begin{tabular}{|c|c|c|}
\hline ASTM N $^{\circ}$ & $\mathrm{mm}$ & Porcentaje en peso que pasa \\
\hline $3 / 8 "$ & 9.51 & 100 \\
\hline 4 & 4.76 & 94.5 \\
\hline 8 & 2.38 & 69.5 \\
\hline 16 & 1.19 & 54.2 \\
\hline 30 & 0.595 & 39.4 \\
\hline 50 & 0.297 & 16.1 \\
\hline 100 & 0.149 & 4.4 \\
\hline
\end{tabular}

Tabla 5. Características físicas del árido fino natural

\begin{tabular}{|l|c|}
\hline Propiedades \\
\hline Densidad aparente compactada $\left(\mathrm{Kg} / \mathrm{dm}^{3}\right)$ & 1.7 \\
\hline Densidad aparente suelta $\left(\mathrm{Kg} / \mathrm{dm}^{3}\right)$ & 1.59 \\
\hline Densidad real seca $\left(\mathrm{Kg} / \mathrm{dm}^{3}\right)$ & 2.59 \\
\hline Densidad real saturada sup. seca $\left(\mathrm{Kg} / \mathrm{dm}^{3}\right)$ & 2.65 \\
\hline Densidad neta $\left(\mathrm{Kg} / \mathrm{dm}^{3}\right)$ & 2.74 \\
\hline Absorción (\%) & 2 \\
\hline Partículas menores a $0.08 \mathrm{~mm} \mathrm{( \% )}$ & 1.5 \\
\hline Impurezas orgánicas & 2 \\
\hline
\end{tabular}

Tabla 6. Características granulométricas árido grueso reciclado

\begin{tabular}{|c|c|c|}
\hline ASTM No $^{\circ}$ & $\mathrm{mm}$ & Porcentaje en peso que pasa \\
\hline $1 / 2 "$ & 12.7 & 100 \\
\hline $3 / 8 "$ & 9.51 & 62.7 \\
\hline 4 & 4.76 & 21.3 \\
\hline 8 & 2.38 & 16.1 \\
\hline
\end{tabular}

\section{PRUEBAS DE LABORATORIO}

\section{Dosificaciones}

Para calcular las proporciones de los distintos materiales utilizados en las mezclas se utilizó el método de dosificación Faury - Joisel (Zabaleta, 1996), que se basa en determinar las proporciones de los áridos en base al mejor ajuste a una curva granulométrica de referencia, en la que se considera al cemento como un elemento más de la mezcla. Las características de las mezclas utilizadas para elaborar los bloques con áridos naturales y áridos reciclados se ilustran en la Tabla 8. La notación de la mezcla indica los dos tipos de mezclas confeccionadas (AN, para los bloques compuestos solamente de áridos naturales y AR, para los bloques compuestos con árido grueso reciclados y árido fino natural), ambos tipos de mezclas fueron diseñadas para obtener una resistencia de compresión cúbica de $15 \mathrm{MPa}$ a los 28 días (probetas cúbicas de $200 \mathrm{~mm}$ de arista), con un $90 \%$ de confianza, un tamaño máximo de $12.5 \mathrm{~mm}$ y asentamiento igual a $0 \mathrm{~cm}$. según Nch 1019 E Of.74.

\section{Fabricación de los bloques de hormigón}

El bloque escogido para someterlo a este estudio es el codificado como clase A según la NCh 181 Of 67 , de $140 \pm 3 \mathrm{~mm}$ de ancho, $190 \pm 3 \mathrm{~mm}$ de alto y $390 \pm 3 \mathrm{~mm}$ de largo. El cemento y los áridos se mezclaron en seco, hasta que adquirieron un color uniforme. Luego se les añadió el agua y el conjunto se mezcló durante 1,5 minutos en la mezcladora mecánica. Posteriormente, la mezcla obtenida se introdujo en un molde metálico para luego ser compactada en una mesa vibradora marca 
Control, modelo C 158, con una frecuencia de 3.000 r.p.m.. Una vez que comenzaba a aparecer el agua en la cara superior de la mezcla se procedía a desmoldar cada uno de los bloques confeccionados, como se ilustra en la Fig. 1.

Tabla 7. Características físicas del árido grueso reciclado

\begin{tabular}{|l|l|}
\hline Propiedades \\
\hline Densidad aparente compactada $\left(\mathrm{Kg} / \mathrm{dm}^{3}\right)$ & 1.43 \\
\hline Densidad aparente suelta $\left(\mathrm{Kg} / \mathrm{dm}^{3}\right)$ & 1.34 \\
\hline Densidad real seca $\left(\mathrm{Kg} / \mathrm{dm}^{3}\right)$ & 2.47 \\
\hline Densidad real saturada sup. seca $\left(\mathrm{Kg} / \mathrm{dm}^{3}\right)$ & 2.55 \\
\hline Densidad neta $\left(\mathrm{Kg} / \mathrm{dm}^{3}\right)$ & 2.67 \\
\hline Absorción (\%) & 3.03 \\
\hline Partículas menores a $0.08 \mathrm{~mm} \mathrm{( \% )}$ & 1.71 \\
\hline
\end{tabular}

Tabla 8. Dosificación en Kg. para $1 \mathrm{~m}^{3}$ de mezcla

\begin{tabular}{|l|c|l|c|}
\hline \multicolumn{2}{|c|}{ AN H-15 9012.50} & \multicolumn{2}{c|}{ AR - H-15 90 12.5 0 } \\
\hline Árido grueso natural & 830 & Árido grueso reciclado & 833 \\
\hline Árido fino natural & 1170 & Árido fino natural & 1004 \\
\hline Cemento ARI & 240 & Cemento ARI & 240 \\
\hline Agua & 168 & Agua & 180 \\
\hline
\end{tabular}

Los bloques ya desmoldados se curaron en una sala a temperatura ambiente durante 6 horas, luego se ingresaron a un sala de curado a vapor, a una temperatura promedio de $20^{\circ} \mathrm{C}$, por 14 días. El proceso de curado finalizó en una sala a temperatura ambiente hasta los 28 días.

\section{Ensayos realizados}

Los ensayos realizados a los bloques de hormigón según la NCh 182 of 55 conducen a determinar la calidad de los bloques destinados a construcciones por medio de la verificación de su resistencia mecánica a la compresión (Fig. 2), absorción de máxima de agua y contenido de humedad. Los ensayos de compresión de bloques se realizaron con una prensa marca Control, modelo C-6222 con una capacidad de carga máxima de $3000 \mathrm{KN}$, de acuerdo a la norma NCh 182. Of. 55. Se obtuvo el promedio de 5 bloques por cada tipo de mezcla, los cuales fueron ensayados a los 7, 14 y 28 días. La absorción máxima de agua y el contenido de humedad, se realizaron para cada tipo de bloques, los compuestos únicamente por áridos naturales y los que contenían su fracción gruesa de áridos reciclados, de acuerdo a lo señalado en la normativa.

\section{RESULTADOS Y DISCUSION}

La Fig. 3 muestra el resultado del promedio de las resistencias a la compresión a los 7, 14 y 28 días de los bloques confeccionados completamente con árido natural y los confeccionados solo con la fracción gruesa de árido reciclado su mezcla.

Es posible apreciar que ambos bloques cumplieron con las resistencias exigidas por normativa NCh 181of 67, demostrando que el promedio de resistencias obtenidas a la compresión debe ser superior a los $45 \mathrm{~kg} / \mathrm{cm}^{2}$ para el promedio de las 5 muestras a los 28 días, además la Fig. 3 ilustra la disminución de resistencia a la compresión de un $15 \%$ en los bloques confeccionados con árido reciclado bajo las mismas condiciones que los bloques confeccionados completamente por áridos naturales. 


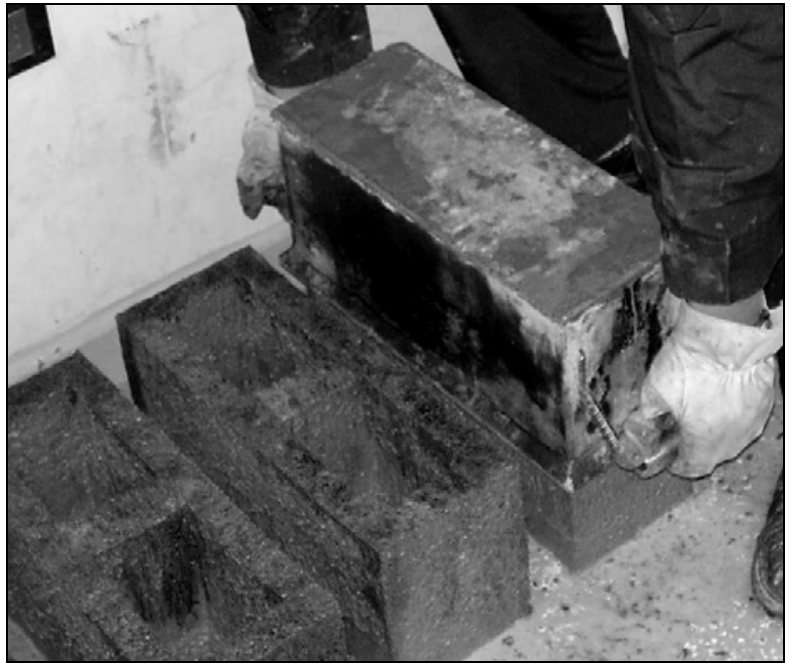

Fig. 1: Bloque de hormigón recién desmoldado.

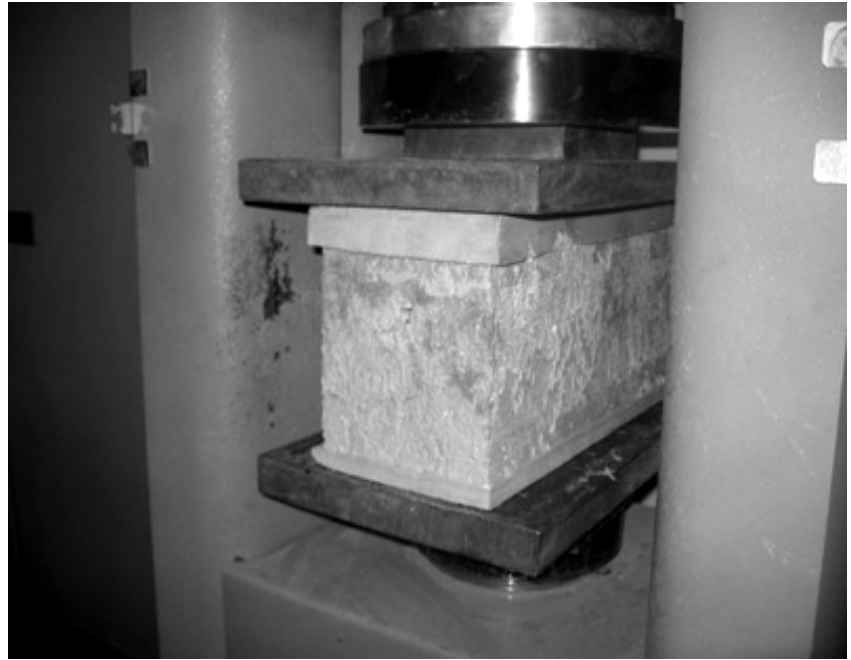

Fig. 2: Bloque sometido a ensayo de compresión.

Topcu y Guncan (1995) Ilegaron a conclusiones similares en probetas de hormigón, utilizando diferentes porcentajes de adiciones del $0,30,50,70$ and 100 por ciento de árido reciclado en hormigones de prueba y obtuvieron una baja en los esfuerzos de compresión a medida que aumentaban los porcentajes de agregados reciclados. Por otra parte, Rakshvir y Barai (2006) concluyen que las resistencias a la compresión del hormigón decrecen hasta un $10 \%$ en función del aumento de agregados reciclados en la mezcla de hormigón.

De acuerdo a lo anterior y lo expuesto por Poon, et al. (2002), es posible afirmar que la adición de material granular reciclado en las mezclas de hormigón causa una perdida de resistencia a la compresión en bloques de hormigón, no tan significativa que impida el uso de este material, con la restricción de que se deben considerar adiciones inferiores a un 50\% de agregado reciclado en la mezcla para evitar problemas de trabajabilidad de la mezcla y resistencia en los bloques. Se puede establecer que la metodología de diseño de mezclas de hormigón propuesta por Faury-Joisel utilizada para el diseño de bloques de hormigón, es adecuada, debido a que entrega proporciones de árido grueso reciclado, acorde a lo postulado anteriormente; para el caso de las pruebas realizadas fue de un $36 \%$ en peso, respecto al resto de los componentes de la mezcla.

La Fig. 4 ilustra las densidades promedios de los bloques confeccionados. Los bloques compuestos por áridos reciclados muestran un menor peso por unidad que los confeccionados con árido normal, estos valores se deben principalmente a que el árido reciclado grueso tiene densidades inferiores respecto al árido normal grueso utilizado, como se ilustró el las Tablas 3 y 7 . Esto concuerda con lo determinado por Topcu y Sengel (2004), donde muestran que la adición de árido reciclado es proporcional a la disminución de la densidad.

La absorción de agua ilustrada en la Tabla 9 para los bloques reciclados cumplen con la exigencia de la Norma NCh 181 Of 67, muy por debajo de los $210 \mathrm{~kg}$ agua $/ \mathrm{m} 3$ de hormigón exigida. Además, muestra una pequeña diferencia de absorción en relación a bloques construidos con áridos naturales, que se explica por la mayor absorción de agua de los áridos reciclados como se ilustra en las Tablas 3 y 7.

Tabla 9. Absorción de bloques

\begin{tabular}{|c|c|c|c|}
\hline \multicolumn{2}{|c|}{$\begin{array}{c}\text { Bloque } \\
\text { AN H-15 90 12.5 0 }\end{array}$} & \multicolumn{2}{c|}{ Bloque } \\
AR $-\mathrm{H}-159012.50$ \\
\hline \multicolumn{2}{|c|}{ Absorción } & \multicolumn{2}{|c|}{ Absorción } \\
\hline (kg agua/ $\mathrm{m}^{3}$ hormigón) & $(\%)$ & $\left(\mathrm{kg}\right.$ agua/ $\mathrm{m}^{3}$ hormigón $)$ & $(\%)$ \\
\hline 126.31 & 5.6 & 141.34 & 6.7 \\
\hline
\end{tabular}




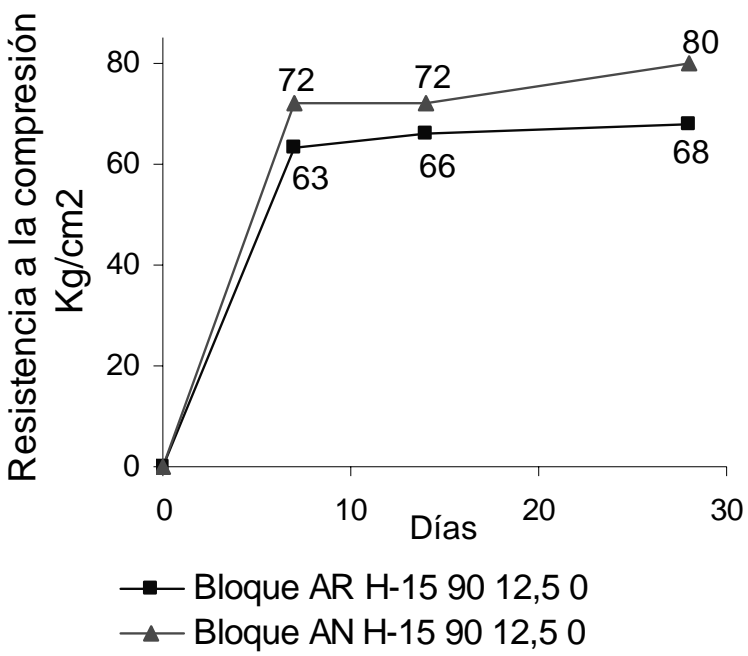

Fig.3. Resistencias promedio de Bloque sometido Fig. 4: Densidades de Bloques confeccionados.

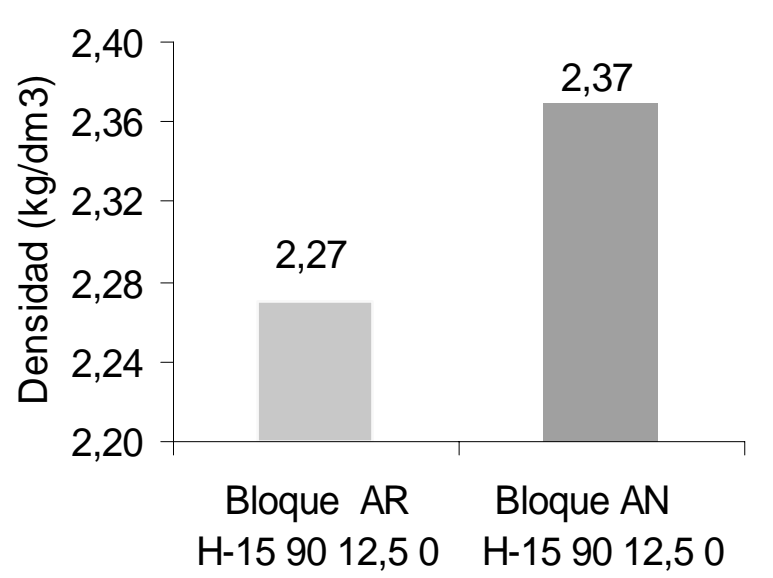

El contenido de humedad resultante para los bloques ensayados está referido a la absorción máxima de cada tipo de bloque. A diferencia de las pruebas anteriores, esta cualidad de los bloques depende directamente con las condiciones de almacenamiento de los bloques. Los bloques ensayados se almacenaron al aire libre bajo condiciones climáticas desfavorables y se obtuvieron contenidos de humedad del $90 \%$ para ambos tipos de bloques.

\section{CONCLUSIONES}

El estudio realizado muestra una técnica desarrollada para la producción de bloques de hormigón con áridos reciclados. Las pruebas de laboratorio realizadas, tanto a los áridos reciclados como a los bloques de hormigón compuestos por éstos, demuestran la factibilidad técnica de que bloques de hormigón diseñados con el método de dosificación de mezclas de hormigón propuesto por FauryJoisel y confeccionados con áridos reciclados puedan ser utilizados como elementos constructivos estructurales al cumplir los estándares exigidos por la normativa, colaborando con esta solución, a los problemas medioambientales provocados por la extracción de áridos y la generación de residuos producto de la actividad construcción.

\section{AGRADECIMIENTOS}

Los autores agradecen al Gobierno Regional de la Araucanía por el financiamiento de esta investigación a través del Fondo Nacional para el Desarrollo Regional. Asimismo los autores agradecen la donación de materiales y cooperación de la empresa cementos Bío-Bío.

\section{REFERENCIAS}

ASTM C-595, American Society for Testing and Materials, Standard Specification for Blended Hydraulic Cements, West Conshohocken, PA, (2003).

Aguilar, C., Muñoz, M.P. y Loyola O., Uso de hormigón reciclado para la fabricación de hormigones, Revista Ingeniería de Construcción 20 (1), pp. 35-44, (2005).

BS EN 197-1:2000, British-Adopted European Standard, Cement. Composition, specifications and conformity criteria for common cements, pp. 1-53, (2000).

NCh 181 Of. 67, Norma Chilena Oficial, Bloques huecos de hormigón de cemento, pp. 1-5, Santiago, Chile, (1967). 
NCh 182. Of. 55, Norma Chilena Oficial, Ensayos de bloques de hormigón, pp. 1-5, Santiago, Chile, (1955).

Montoya, H.D., Pino C.L. y Valdés G.A., Reutilización de residuos del hormigón, BIT 12 (41), pp. 4447, (2005).

Moreno, H. y Varela, J., Geología, volcanismo y sedimentos piroclásticops cuaternarios de la Región Central y Sur de Chile. En Suelos volcánicos de Chile (Tosso, J.; editor). Instituto de Investigaciones Agropecuarias, Capítulo 6, p. 491-526. Santiago, Chile (1985).

Poon,C.S., Kou,S.C. y Lam,L., Use of recycled aggregates in molded concrete bricks and blocks, Construction and Building Materials 16 (5), pp. 281-289, (2002).

Poon, C.S., Yu, A.T.W. y Ng, L.H., On-site sorting of construction and demolition waste in Hong Kong, Resources, Conservation and Recycling 32 (2), pp. 157-172, (2001).

Rakshvir, M. y Barai, S.V., Studies on recycled aggregates-based concrete, Waste Management and Research 24 (3), pp. 225-233 (2006).

Sánchez, M. y Alaejos, P., Influencia del árido reciclado en las propiedades del hormigón estructural, Cemento y Hormigón (889), pp. 54-61, (2006).

Topcu, I.B., Physical and mechanical properties of concretes produced with waste concrete, Cement and Concrete Research 27 (12), pp. 1817-1823 (1997).

Topcu, I.B. y Sengel, S., Properties of concretes produced with waste concrete aggregate, Cement and Concrete Research 34 (8), pp. 1307-1312 (2004).

Topcu, I. B. y Guncan, N. F., Using waste concrete as aggregate, Cement and Concrete Research 25 (7), pp. 1385-1390 (1995).

Zabaleta H., Compendio de tecnología del hormigón, $2^{\mathrm{a}}$ edición, Publicaciones Instituto Chileno del Hormigón, pp. 85-90, Santiago, Chile (1996). 